\title{
Inovação em Logística e Operações: Um Estudo Biblimétrico sobre Gestão de Estoques no Brasil
}

\author{
Arthur Antonio Silva Rosa \\ Mestrado em Administração pela Faculdade de Gestão e Negócios da Universidade \\ Federal de Uberlândia - UFU \\ Administrador \\ Av. João Naves de Ávila, 2121. Santa Mônica. Uberlândia/MG. CEP: \\ 38408-014 \\ E-mail:arthurasr@hotmail.com \\ Thalita Emanuelle Farias Bastos \\ Mestrado Profissional em Administração pela Faculdade de Gestão e Negócios da \\ Universidade Federal de Uberlândia - UFU \\ Administradora \\ Av. João Naves de Ávila, 2121. Santa Mônica. Uberlândia/MG. CEP: \\ 38408-014 \\ E-mail: thalita.bastos.adm@gmail.com
}

Brunno da Silva Mestrado em andamento em Administração pela Faculdade de Gestão e Negócios da Universidade Federal de Uberlândia - UFU

Administrador Av. João Naves de Ávila, 2121. Santa Mônica. Uberlândia/MG. CEP: 38408-014

E-mail:drbrunnos@gmail.com

Kárem Cristina de Sousa Ribeiro Pós-Doutorado em Administração pela Faculdade de Economia, Administração, Contabilidade e Atuária - FEA/USP Professora da Universidade Federal de Uberlândia - UFU Av. João Naves de Ávila, 2121. Santa Mônica. Uberlândia/MG. CEP: 38408-014 E-mail:kribeiro@ufu.br

\section{RESUMO}

No contexto da inovação empresarial, a gestão do estoque tem se destacado em virtude da sua importância para a lucratividade, retorno sobre o capital investido e como estratégia para suportar a definição de vantagem competitiva para a organização. Diante desse cenário, o objetivo desta pesquisa é identificar as principais características dos artigos publicados sobre gestão de estoques e inovação no Brasil entre 2010 e 2018, sob o ponto de vista do método de controle abordado. Para se atingir o objetivo, utilizou-se como metodologia a pesquisa bibliométrica com abordagem quantitativa e a coleta de dados foi realizada através da plataforma SPELL. Os principais resultados 
Inovação em Logística e Operações: Um Estudo Biblimétrico sobre Gestão de Estoques no Brasil

Arthur Antonio Silva Rosa, Thalita Emanuelle Farias Bastos, Brunno da Silva, Kárem Cristina de Sousa

Ribeiro

demonstram que a maioria dos artigos sobre gestão de estoques foram publicados em 2016, possuem dois autores, a Instituição de Ensino que mais autores estão afiliados foi a USP, os métodos mais abordados foram o LEC, Just in time e Curva ABC que abordam a gestão de estoque de forma tradicional sem acréscimo de inovação. Já os que abordam aspectos inovadores, estão relacionados à tecnologia da informação. $\mathrm{O}$ estudo contribuiu para a definição do perfil de publicações sobre o tema, auxiliando na definição de trabalhos futuros.

Palavras-chave: Gestão de estoques. Métodos. Inovação. Bibliometria.

\section{Logistics and Operations Innovation: A Bibliometric Study on Inventory Management in Brazil}

\section{ABSTRACT}

In the context of business innovation, inventory management has stood out due to its importance for profitability, return on invested capital and as a strategy to support the definition of competitive advantage for the organization. Given this scenario, the objective of this research is to identify the main characteristics of articles published on inventory management and innovation in Brazil between 2010 and 2018, from the point of view of control methods. To achieve the objective, a bibliometric research within a quantitative approach was carried out, and data collection was performed through the SPELL platform. The main results demonstrate that most articles on inventory management were published in 2016, have two authors, the Educational Institution that most authors are affiliated in are USP, the most discussed methods are LEC, Just in time and $A B C$ Curve and approach inventory management in a traditional way, without adding innovation. Those that address innovative aspects are related to information technology. The study contributed to the definition of the profile of publications on the topic, helping to define future works.

Keywords: Inventory management. Methods. Innovation. Bibliometrics.

\section{INTRODUÇÃO}

O aumento da economia global, a concorrência e a tecnologia exigem cada vez mais agilidade e flexibilidade das organizações. A exploração de diferentes estratégias que possibilitem melhorias em processos produtivos e seus controles é uma prática utilizada pelas organizações para conseguir vantagens em relação aos seus 
Inovação em Logística e Operações: Um Estudo Biblimétrico sobre Gestão de Estoques no Brasil

Arthur Antonio Silva Rosa, Thalita Emanuelle Farias Bastos, Brunno da Silva, Kárem Cristina de Sousa

Ribeiro

competidores. Um dos instrumentos para eficiência da cadeia produtiva nas organizações modernas é a gestão de estoque, que constitui um tema clássico na engenharia de produção (Davis, Chase, \& Aquilano, 2001; Stevenson, 2001; Corrêa, Gianesi, \& Caon, 2001; Moreira, 2008).

A gestão de estoques é diferente da administração de outros ativos e obrigações. Esses ativos têm um teor físico, o que não se igualam aos ativos puramente financeiros. Os estoques representam custos significativos para as empresas, e sua gestão eficiente torna-se fator essencial de competitividade (Scherr, 1989). Arnold (2001) mencionou a importância na gestão para otimização dos estoques quando demonstrou que eles podem representar de $20 \%$ a $60 \%$ dos ativos no balanço de uma organização. Se por um lado os estoques em excesso podem onerar os custos da empresa, por outro lado, a sua falta pode ocasionar tanto paradas na produção como redução do nível de serviço aos clientes.

Cabe ressaltar que o estudo de modelos de gestão de estoques normalmente não é simples, dada a presença de incertezas, isto é, variáveis que não podem ser preditas antecipadamente e que precisam ser estimadas de alguma forma com intuito de maximizar ou minimizar uma função objetivo, relacionada à qualidade da gestão, levando em conta a estratégia adotada (Graither \& Frazier, 2001).

Algumas pesquisas bibliométricas com a temática de gestão de estoque já foram desenvolvidas recentemente no Brasil. Scheidegger, Favaretto e Lima (2013) analisaram os estudos publicados entre 2004 e 2013 que abordavam a gestão de estoque e políticas de reposição aos quais foram desenvolvidas para o setor público. Longaray, Tondolo, Ávila, Munhoz, Tondolo e Sarquis (2017) analisaram a produção científica brasileira de 2006 a 2015 sobre a gestão de estoque no varejo. Ainda, Silveira, Eduardo, Ribeiro, Chaebo e Nóbrega (2018) ao analisarem a produção científica no Portal de Periódicos da Capes sobre a gestão de estoque, identificaram a maior incidência de pesquisas empíricas com abordagem de metodologias quantitativas e ausência de artigos com maior qualificação Qualis A1. 
No entanto, os estudos não destacaram os métodos de controle de estoque que apresentavam algum grau de inovação, o que está sendo abordado na presente pesquisa. Diante desse contexto, a questão que norteou o desenvolvimento do trabalho foi: Quais as principais características dos estudos científicos brasileiros publicados de 2010 a 2018 a respeito da gestão de estoques e quais abordam os métodos inovadores? Com isso, o objetivo é realizar uma análise bibliométrica dos artigos publicados no Brasil no período de 2010 a 2018 sobre a temática Gestão de Estoque e Inovação na base de conhecimento da SPELL, sob o ponto de vista do método de controle abordados pelos autores.

Como consequência, espera-se que os resultados possam indicar as tendências inovadoras na gestão de estoque que podem auxiliar os gestores na tomada de decisões em relação ao controle do inventário. Para o meio acadêmico, a pesquisa contribuirá para demonstrar a evolução das pesquisas nessa temática.

Além desta introdução, a pesquisa está estruturada da seguinte forma: na segunda seção expõe-se as referências teóricas que dão embasamento ao tema abordado; na terceira seção, descreve-se a metodologia aplicada; na quarta seção, apresentam-se e analisam-se os resultados da pesquisa bibliométrica; e, por fim, apresentam-se as considerações finais.

\section{GESTÃO DE ESTOQUE}

A gestão de estoque é uma das mais velhas preocupações na ciência da gestão e, diferentemente dos demais ativos e passivos, o estoque possui natureza mais física do que puramente financeira (Scherr, 1989). Essa preocupação pode estar ligada a sua participação no total de ativos pela importância na gestão do ciclo operacional ou pelo impacto na lucratividade e retorno sobre o capital investido (Weston \& Brigham, 2000; Assaf Neto, 2007). "Gerir os níveis de estoques é fundamental para estabelecer uma vantagem competitiva a longo prazo" (Hansen \& Mowen, 2001, p. 737). 
Inovação em Logística e Operações: Um Estudo Biblimétrico sobre Gestão de Estoques no Brasil

Arthur Antonio Silva Rosa, Thalita Emanuelle Farias Bastos, Brunno da Silva, Kárem Cristina de Sousa

Ribeiro

Segundo Assaf Neto e Silva (2007), os motivos para se estocar se resumem basicamente em tornar o fluxo econômico contínuo para não paralisar a produção ou perder vendas; características econômicas particulares de cada setor; perspectiva de um aumento imediato no custo do produto (acredita-se que o ganho obtido pela compra antecipada compense os custos de estocagem); proteção contra perdas inflacionárias; e política de vendas do fornecedor (concessão de descontos ao adquirir maiores quantidades). Ballou (2006) acrescenta a melhoria do serviço ao cliente e redução de custos (mesmo que ocorram maiores custos de manutenção, há uma redução de custos operacionais em outras atividades do canal de suprimentos).

Para elaborar uma estratégia de inventário, conforme Scherr (1989), a empresa deve levar em consideração os custos e as características particulares do estoque envolvido. As estratégias de encomenda de materiais são elaboradas com a utilização de modelos matemáticos. Porém, como há incerteza na definição de parâmetros para se fazer as decisões de encomenda, há risco associado a essas estratégias.

Depois de identificar os custos e características do estoque e escolher o método de abordagem, um modelo deve ser desenvolvido para formular a política. Os modelos mais utilizados para gestão do estoque estão resumidos na Figura 1:

\begin{tabular}{|c|c|c|c|}
\hline Modelo & Conceito & Fórmula/Características & Autores \\
\hline $\begin{array}{l}\text { LEC (Lote } \\
\text { Econômico de } \\
\text { Compra) }\end{array}$ & $\begin{array}{l}\text { Representa a } \\
\text { quantidade ótima de } \\
\text { cada pedido de modo } \\
\text { que os custos totais } \\
\text { (de pedido e } \\
\text { estocagem) sejam os } \\
\text { menores possíveis }\end{array}$ & $\begin{array}{l}\qquad Q=\sqrt{\frac{2 F S}{C P}} \\
\text { Onde: } \\
\text { F: custo do pedido } \\
\text { S: quantidade anual necessária } \\
\text { ou de vendas anuais } \\
\text { S: soma dos custos diretamente } \\
\text { proporcionais ao estoque } \\
\text { P: valor por unidade do produto }\end{array}$ & $\begin{array}{l}\text { Scherr (1989), Weston e } \\
\text { Brigham (2000), Hansen } \\
\text { e Mowen (2001), Assaf } \\
\text { Neto (2006), Ballou } \\
\text { (2006), Assaf Neto e } \\
\text { Silva (2007) }\end{array}$ \\
\hline Just in time & $\begin{array}{l}\text { Filosofia de gestão } \\
\text { empresarial baseada } \\
\text { na eliminação total } \\
\text { dos estoques e } \\
\text { produção puxada } \\
\text { pela demanda }\end{array}$ & $\begin{array}{l}\text { Requer muita coordenação entre } \\
\text { o fabricante e seus fornecedores }\end{array}$ & $\begin{array}{l}\text { Weston e Brigham } \\
(2000), \quad \text { Hansen e } \\
\text { Mowen (2001), Assaf } \\
\text { Neto e Silva (2007), } \\
\text { Garrison; } \\
\text { Brewer (2007) Noreen; }\end{array}$ \\
\hline
\end{tabular}


Inovação em Logística e Operações: Um Estudo Biblimétrico sobre Gestão de Estoques no Brasil

Arthur Antonio Silva Rosa, Thalita Emanuelle Farias Bastos, Brunno da Silva, Kárem Cristina de Sousa

Ribeiro

\begin{tabular}{|c|c|c|c|}
\hline $\begin{array}{l}\text { MRP I } \\
\text { (Manufacturin } \\
\text { g Resources } \\
\text { Planning I) }\end{array}$ & $\begin{array}{l}\text { O ponto-chave do } \\
\text { MRP I é determinar } \\
\text { os materiais e outras } \\
\text { exigências a partir da } \\
\text { demanda do cliente }\end{array}$ & $\begin{array}{l}\text { Fundamenta-se, principalmente, } \\
\text { nas informações da lista de } \\
\text { materiais, ciclos de produção de } \\
\text { cada item, programação mestre, } \\
\text { níveis de inventários e no lote } \\
\text { econômico para executar o } \\
\text { planejamento das necessidades } \\
\text { de materiais (quanto e em que } \\
\text { momento) }\end{array}$ & $\begin{array}{l}\text { Norman (1983), Slack } \\
\text { Chambers; Johnston } \\
\text { (2002) }\end{array}$ \\
\hline $\begin{array}{l}\text { MRP II } \\
\text { (Manufacturin } \\
\text { g Resources } \\
\text { Planning II) }\end{array}$ & $\begin{array}{l}\text { Sistema } \\
\text { computacional que } \\
\text { objetiva cumprir os } \\
\text { prazos de entrega } \\
\text { com a formação } \\
\text { mínima de estoques }\end{array}$ & $\begin{array}{l}\text { A principal característica dessa } \\
\text { técnica está no nível de } \\
\text { detalhamento dos itens } \\
\text { planejados, programados e } \\
\text { controlados }\end{array}$ & $\begin{array}{l}\text { Adams e Cox (1985), } \\
\text { Sacomano (1990), Slack } \\
\text { Chambers; Johnston } \\
\text { (2002), Assaf Neto e } \\
\text { Silva (2007) }\end{array}$ \\
\hline $\begin{array}{l}\text { Ponto de } \\
\text { ressuprimento }\end{array}$ & $\begin{array}{lrr}\text { Ponto no tempo } \\
\text { quando um novo } \\
\text { pedido deve ser feito } \\
\text { ou iniciada } \quad \text { a } \\
\text { preparação } \\
\end{array}$ & $\begin{array}{l}\text { Ponto de ressuprimento = taxa } \\
\text { de consumo } \times \text { prazo de entrega }\end{array}$ & $\begin{array}{l}\text { Hansen e Mowen } \\
\text { (2001), Ballou (2006) }\end{array}$ \\
\hline Curva ABC & $\begin{array}{l}\text { Segregação por do } \\
\text { estoque pua } \\
\text { importância r no } \\
\text { faturamento da } \\
\text { empresa (com base } \\
\text { na Lei de Pareto) }\end{array}$ & $\begin{array}{l}\text { A: menor quantidade itens de } \\
\text { maior importância e com grande } \\
\text { impacto sobre o faturamento; } \\
\text { B: importância intermediária; } \\
\text { C: maior quantidade de itens } \\
\text { com menor impacto sobre o } \\
\text { faturamento. }\end{array}$ & $\begin{array}{l}\text { Assaf Neto (2006), } \\
\text { Ballou (2006), Assaf } \\
\text { Neto e Silva (2007) }\end{array}$ \\
\hline
\end{tabular}

\section{Figura 1. Modelos mais utilizados na gestão de estoque}

Fonte: Dados da pesquisa.

Dentre esses métodos, Rogers, Ribeiro e Rogers (2004) destacaram que o Lote Econômico de Compra (LEC) é o mais utilizado dentre os modelos tradicionais. Os autores formularam uma estratégia para cálculo do LEC em situações de risco, utilizando o método da simulação de Monte Carlo. Rosa, Mayerle e Gonçalves (2010) também estudaram situações em que o LEC é adotado como política de reposição, comparando os tempos médios de ressuprimento do fornecedor pelas sistemáticas de revisão contínua e revisão periódica. Foram encontrados resultados que demonstram que a revisão contínua é mais eficiente em termos de estoque médio e custo total. Já Callefi (2019) mostrou em seu estudo que o LEC pode ser definido com base na distribuição normal. 
Inovação em Logística e Operações: Um Estudo Biblimétrico sobre Gestão de Estoques no Brasil

Arthur Antonio Silva Rosa, Thalita Emanuelle Farias Bastos, Brunno da Silva, Kárem Cristina de Sousa

Ribeiro

\subsection{Gestão de estoques na prática}

Gianesi e Biazzi (2011), baseado em suas experiências profissionais como consultores, afirmam que é incomum encontrar empresas brasileiras que utilizam métodos quantitativos formais para apoio à gestão de estoques, mas comum o uso de métodos empíricos, qualitativos e com base na intuição. Em seu trabalho os autores evidenciaram a necessidade de uma abordagem estratégica na gestão dos estoques e propuseram um caminho para alcançar essa abordagem que poderá afetar positivamente o poder de competitividade da empresa.

Independente da forma de controle, seja por mecanismos mais automatizados ou manuais e empíricos, é inegável a importância da gestão de estoque para as empresas conforme destacado por Scherr, 1989; Weston \& Brigham, 2000; Hansen \& Mowen, 2001; Assaf Neto, 2006; Assaf Neto \& Silva 2007. Devido a isso, pesquisadores têm abordado esse tema em seus trabalhos, contribuindo para o fortalecimento de teorias de gestão de estoque e sua aplicação prática, além da disseminação de técnicas e modelos. Na Figura 2, estão resumidos trabalhos nacionais e internacionais sobre a gestão de estoque nas organizações.

\begin{tabular}{|c|c|c|c|}
\hline Título & Autores/Ano & Objetivo & Resultados \\
\hline \begin{tabular}{ll} 
Aversão ao \\
risco no \\
gerenciamento \\
\multicolumn{2}{l}{ de estoque }
\end{tabular} & $\begin{array}{l}\text { Xin Chen, } \\
\text { Melvyn Sim, } \\
\text { David Simchi- } \\
\text { Levi, Peng } \\
\text { Sun (2007) }\end{array}$ & $\begin{array}{l}\text { Propõe uma estrutura } \\
\text { para incorporar a aversão } \\
\text { ao risco em modelos de } \\
\text { inventário multi-período, } \\
\text { bem como modelos multi- } \\
\text { períodos que coordenam } \\
\text { estratégias de estoque e } \\
\text { preços }\end{array}$ & $\begin{array}{l}\text { Os resultados computacionais } \\
\text { demonstram que a política ótima é } \\
\text { relativamente insensível a pequenas } \\
\text { mudanças no nível de aversão ao risco } \\
\text { do tomador de decisão }\end{array}$ \\
\hline $\begin{array}{l}\text { O efeito do } \\
\text { gerenciamento } \\
\text { de estoque no } \\
\text { desempenho } \\
\text { da empresa }\end{array}$ & $\begin{array}{l}\text { Dimitrios P. } \\
\text { Koumanakos } \\
(2008)\end{array}$ & $\begin{array}{l}\text { Testar a hipótese de que } \\
\text { a gestão eficiente } \\
\text { (enxuta) de estoques leva } \\
\text { a uma melhoria no } \\
\text { desempenho financeiro } \\
\text { de uma empresa }\end{array}$ & $\begin{array}{l}\text { Resultados preliminares, obtidos por } \\
\text { regressões lineares cruzadas, revelam } \\
\text { que quanto maior o nível de estoques } \\
\text { preservados, partindo de operações } \\
\text { enxutas por uma empresa, menor a } \\
\text { taxa de retorno }\end{array}$ \\
\hline
\end{tabular}


Inovação em Logística e Operações: Um Estudo Biblimétrico sobre Gestão de Estoques no Brasil

Arthur Antonio Silva Rosa, Thalita Emanuelle Farias Bastos, Brunno da Silva, Kárem Cristina de Sousa

Ribeiro

\begin{tabular}{|c|c|c|c|}
\hline $\begin{array}{l}\text { Gestão de } \\
\text { estoque de } \\
\text { materiais de } \\
\text { baixíssimo giro } \\
\text { considerando } \\
\text { processos } \\
\text { críticos para a } \\
\text { organização }\end{array}$ & $\begin{array}{l}\text { Jener de } \\
\text { Castro Costa } \\
\text { (2009) }\end{array}$ & $\begin{array}{l}\text { Analisar e apresentar } \\
\text { quais são os impactos } \\
\text { para as organizações, } \\
\text { caso seja adotado o novo } \\
\text { modelo proposto para a } \\
\text { gestão de estoques de } \\
\text { materiais de baixíssimo } \\
\text { giro }\end{array}$ & $\begin{array}{l}\text { A implantação do modelo proposto pode } \\
\text { promover o desdobramento da visão } \\
\text { estratégica da organização através da } \\
\text { associação dos processos, níveis de } \\
\text { serviços desejados, equipamentos e } \\
\text { seus respectivos itens de reposição } \\
\text { garantindo assim maior valor agregado } \\
\text { nas decisões operacionais de gestão de } \\
\text { materiais }\end{array}$ \\
\hline $\begin{array}{lr}\text { Cálculo } & \text { do } \\
\text { ponto } & \text { de } \\
\text { pedido } & \\
\text { baseado } & \text { em } \\
\text { previsões } & \text { de } \\
\text { uma política } \\
<q \text { r }> & \text { de } \\
\text { gestão } & \text { de } \\
\text { estoques } & \\
\end{array}$ & $\begin{array}{l}\text { Eduardo } \\
\text { Saggioro } \\
\text { Garcia e } \\
\text { Virgílio José } \\
\text { Martins } \\
\text { Ferreira Filho } \\
(2009)\end{array}$ & $\begin{array}{l}\text { Apresentar uma } \\
\text { nova abordagem para o } \\
\text { cálculo do ponto de } \\
\text { pedido, baseada na } \\
\text { previsão da demanda } \\
\text { para cada período } \\
\text { durante o tempo de } \\
\text { ressuprimento }\end{array}$ & $\begin{array}{l}\text { O novo modelo amplia o escopo e a } \\
\text { aplicabilidade do modelo clássico além } \\
\text { de ter a possibilidade do seu valor variar } \\
\text { ao longo do tempo, se ajustando a } \\
\text { padrões previsíveis da demanda. Tal } \\
\text { atributo agrega flexibilidade ao } \\
\text { ambiente operacional e pode reduzir } \\
\text { custos de carregar estoques e de } \\
\text { demandas não atendidas }\end{array}$ \\
\hline $\begin{array}{l}\text { Uma } \\
\text { abordagem de } \\
\text { controle de } \\
\text { processo para } \\
\text { gerenciamento } \\
\text { de estoque } \\
\text { tático } \quad \text { em } \\
\text { sistemas de } \\
\text { inventário de } \\
\text { produção }\end{array}$ & $\begin{array}{l}\text { Jay D. } \\
\text { Schwartz e } \\
\text { Daniel E. } \\
\text { Rivera (2010) }\end{array}$ & $\begin{array}{l}\text { Apresenta uma } \\
\text { abordagem fundamental, } \\
\text { porém prática, da } \\
\text { aplicação de princípios de } \\
\text { teoria de controle a um } \\
\text { problema de } \\
\text { gerenciamento de } \\
\text { estoque tático em um } \\
\text { sistema de inventário de } \\
\text { produção }\end{array}$ & $\begin{array}{l}\text { Demonstra-se que uma política de } \\
\text { controle de modelo interno (IMC) com } \\
\text { vários graus de liberdade é adequada } \\
\text { para ajustar o início da fábrica na } \\
\text { presença de mudanças na meta de } \\
\text { estoque, mudanças previstas na } \\
\text { demanda do cliente e mudanças } \\
\text { estocásticas na demanda. A política do } \\
\text { modelo de controle preditivo (MPC) } \\
\text { exibe desempenho equivalente, mas } \\
\text { incorpora a funcionalidade adicional de } \\
\text { gerenciamento de estoque na presença } \\
\text { de restrições e considerações práticas } \\
\text { importantes }\end{array}$ \\
\hline
\end{tabular}

Figura 2. Resumo de trabalhos nacionais e internacionais sobre gestão de estoque

Fonte: Dados da pesquisa.

O desenvolvimento de modelos mais ajustados à realidade da empresa e que contribuem para uma gestão eficiente do estoque é uma metodologia recorrente em pesquisas científicas, através da utilização de ferramentas analíticas. Como resultado, as empresas conseguem agregar valor nas decisões operacionais de gestão de materiais (Costa, 2009) e até reduzir custos em relação ao inventário (Garcia e Ferreira, 2009; Pinto, Tortato, Veiga e Catapan, 2013). 
Inovação em Logística e Operações: Um Estudo Biblimétrico sobre Gestão de Estoques no Brasil

Arthur Antonio Silva Rosa, Thalita Emanuelle Farias Bastos, Brunno da Silva, Kárem Cristina de Sousa

Ribeiro

\subsection{Inovação na gestão de estoques}

O ambiente competitivo exige que as empresas não apenas atendam às necessidades dos clientes com mais precisão, mas também capturem valor fornecendo continuamente novos produtos e serviços (Teece, 2010). Como destacado por Chesbrough (2010, p. 356), uma empresa deve ter "pelo menos o mesmo valor a ganhar com o desenvolvimento de um novo modelo de negócio inovador, a partir do desenvolvimento de uma nova tecnologia inovadora". A necessidade de mudança pode ser em resposta ou antecipação proativa de mudança exógena (Spieth, Schneckenberg, \& Matzler, 2016).

Nesse ambiente, uma forma de obter vantagem competitiva é através da inovação, o que inclui desenvolvimento tecnológico, estratégias de marketing e novas formas de trabalho. E as empresas precisam ter uma visão holística e integrativa sobre a gestão da inovação, pois além de adquirir e utilizar as tecnologias disponíveis, devese identificar e desenvolver competências essenciais para gerar uma estrutura e processo de aprendizado organizacional. Isso contribui para o aperfeiçoamento interno e o desenvolvimento sustentável da empresa (Tidd e Bessant, 2018; Cohen \& Levinthal, 1990).

No ambiente acadêmico, a inovação vem sendo estudada como processo ou como resultado em diversas disciplinas: administração empresarial, ciências econômicas, tecnologia, engenharias, entre outras (Baregheh, Rowley, \& Sambrook, 2009).

Algumas pesquisas aliaram inovação à gestão de estoque, como o trabalho desenvolvido por Librantz, de Araújo, Alves e Pileggi (2010). Os autores utilizaram algoritmos de busca heurística para a definição de parâmetros relacionados a incertezas nos modelos de estoques. Usualmente, esses parâmetros são definidos por técnicas matemáticas, porém em virtude das hipóteses assumidas em situações reais, os modelos ficam fora da realidade. O uso de algoritmos de busca heurística resolve esse problema. Como resultado, constataram que quando os espaços amostrais para 
Inovação em Logística e Operações: Um Estudo Biblimétrico sobre Gestão de Estoques no Brasil

Arthur Antonio Silva Rosa, Thalita Emanuelle Farias Bastos, Brunno da Silva, Kárem Cristina de Sousa

Ribeiro

estimação dos parâmetros são extensos, o uso de algoritmos heurísticos é mais apropriado do que os métodos tradicionais.

Outro estudo inovador na área de gestão de estoque foi desenvolvido por Herbon, Levner e Cheng (2014). Os autores realizaram uma pesquisa abordando o gerenciamento de estoque perecível com preços dinâmicos usando indicadores de tempo e de temperatura vinculados a dispositivos de detecção automática.

Com a evolução constante e impactante da tecnologia, acredita-se que processos, métodos e práticas de gerenciamento de estoque serão aperfeiçoados a cada dia, contribuindo para a inovação na gestão do inventário. Desde os anos 2000, a tecnologia da informação tem sido utilizada para facilitar diversos processos e controles na gestão da cadeia de suprimentos, dentre os quais a gestão do inventário. Tecnologias como identificação por radiofrequência, sistemas integrados de gestão, leitor de código de barras, intercâmbio eletrônicos de dados, dentre outros possibilitavam o processo de um volume maior de dados de diversas fontes com maior precisão e menor tempo (Maçada, Feldens, \& Santos, 2007). Com o advento para a inteligência artificial, as informações e processos passam a ser ainda mais automatizados, o que permite maior agilidade, eficiência e menor custo na gestão de estoques (Lee, Lv, Ng, Ho, \& Choy, 2018).

\section{METODOLOGIA}

O presente artigo tem como objetivo definir as características das publicações brasileiras sobre gestão de estoques nos últimos 9 anos, sob o ponto de vista da inovação e dos métodos utilizados. O estudo possui caráter exploratório, conforme Gil (2007), pois tratou da triagem de publicações entre 2010 e 2018 para a realização de estudo bibliométrico, bem como identifica os índices e as tendências das áreas de publicação, quanto aos diferentes modelos de gestão de estoque.

A bibliometria possui um papel fundamental na análise da produção científica, pois os dados obtidos podem revelar uma mudança de comportamento de uma 
determinada área da ciência. Dessa forma, o estudo bibliométrico também permite uma discussão sobre os aspectos de determinada área do conhecimento e indicando tendências da mesma, e assim, contribuindo para o desenvolvimento de novas pesquisas (Okubo, 1997; Ferreira, 2002).

A pesquisa bibliométrica foi realizada na plataforma SPELL® - Scientific Periodicals Electronic Library, que possui os principais periódicos nas áreas de Administração Pública e de Empresas, Contabilidade e Turismo, indexados em sua base de dados.

A coleta de dados foi realizada em maio de 2019 e, nos campos da plataforma, buscou-se pelos termos "gestão de estoques" no título do documento, resumo e palavras-chave. Também foram inseridos os métodos de gestão de estoques mais comuns na literatura nos campos de busca a fim de aumentar a abrangência da amostra: "MRPI", "MRPII", "Lote Econômico de Compra", "Ponto de Ressuprimento", "Curva ABC" e "Just in time". Foram selecionados os campos: artigos, áreas do conhecimento Administração, Contabilidade e Economia e, por fim todos, os idiomas disponíveis.

Após a realização da busca, obteve-se um total de 33 artigos, sendo retirados 10 artigos que não estavam relacionados com gestão de estoques, resultando no total de 23 artigos. Para a tabulação e análise dos dados, utilizou-se o software Microsoft Office Excel.

As categorias de análise foram definidas com base nas leis de Lotka, Bradford e Zipf, que são consideradas as principais leis da bibliometria (Okubo, 1997; Araújo, 2006). Dessa forma, utilizou-se as seguintes categorias, como a quantidade de autores por publicação, Instituição de Ensino Superior (IES), periódico que o artigo foi publicado, métodos de gestão de estoques e gestão de estoques e inovação. 


\section{RESULTADOS E DISCUSSÃO}

Nesta seção serão apresentados e analisados os principais resultados encontrados a partir da pesquisa bibliométrica.

Com base na Figura 3, observa-se que os anos 2011, 2013, 2015 e 2016 foram os anos que apresentaram o maior número de publicações sobre gestão de estoques, com um total de 4 publicações nos anos citados. No ano de 2014 não houve publicações sobre o tema.

A partir de 2016, nota-se uma queda acentuada na quantidade de publicações, tendo em vista que o somatório das publicações em 2017 e 2018 é a menor quantidade em dois anos consecutivos no período analisado. E a sequência com o maior número de publicações sobre o tema, em 2015 e 2016, pode estar associado à crise econômica do Brasil, onde a otimização dos processos de gestão de estoque se tornam ainda mais relevantes para viabilizar a sobrevivência das empresas.

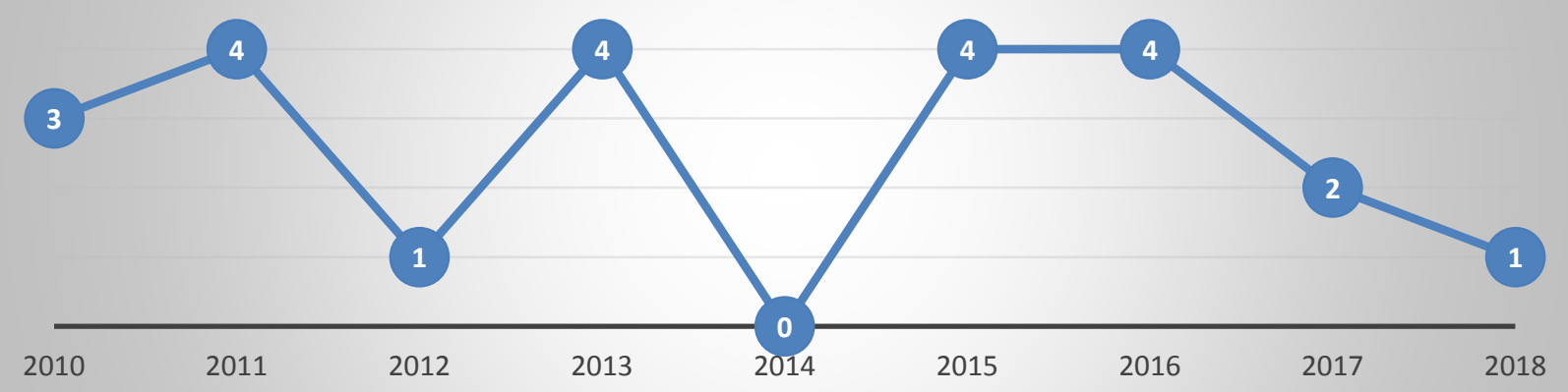

Figura 3. Quantidade de publicações por ano

Fonte: Dados da pesquisa.

A Figura 4 apresenta a quantidade de autores por artigo. Cerca de $42 \%$ dos artigos presentes no período em análise são compostos por dois autores. Trabalhos compostos por 4 autores é o segundo grupo mais comum, com 29\% das publicações no período. Os outros 29\% das publicações são compostas por 3 autores (17\%) e 1 autor 
(12\%). Dessa forma, a maior parte da produção científica sobre gestão de estoque (55\%), é composta por 1 ou 2 autores, o que pode indicar um baixo número de parcerias interinstitucionais, por exemplo.

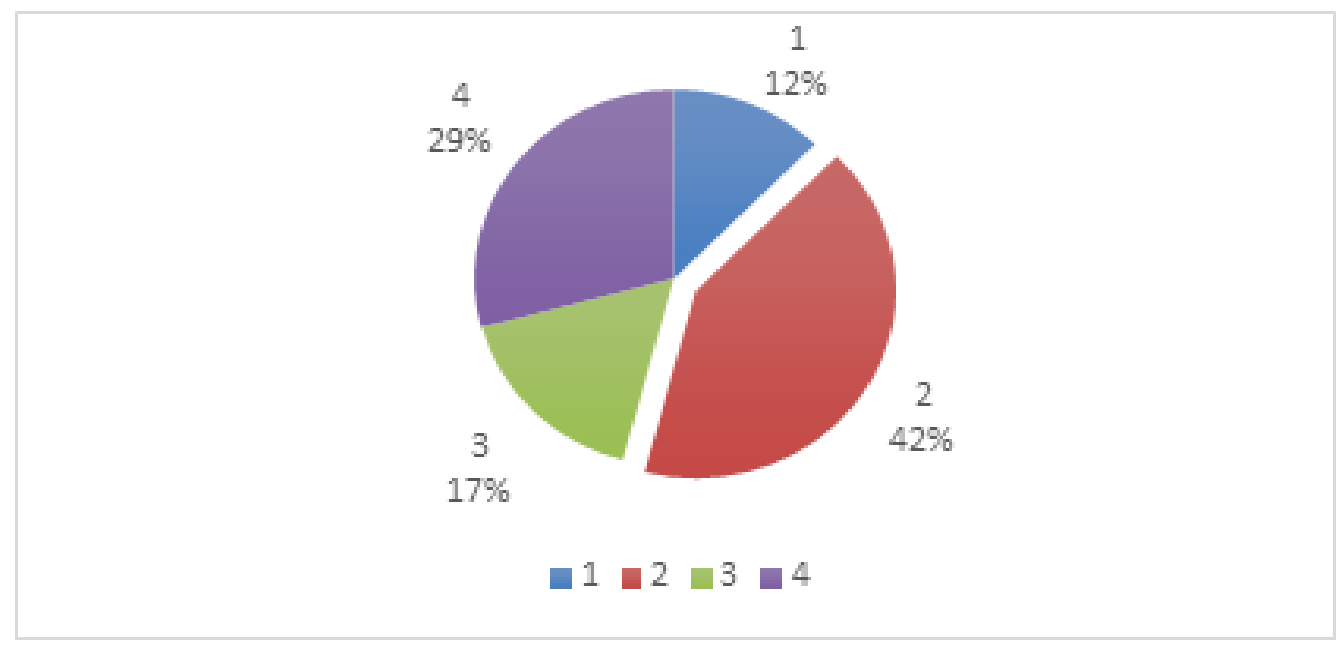

Figura 4. Quantidade de autores por artigo

Fonte: Dados da pesquisa.

Conforme apresentado na Figura 5, a Instituição de Ensino Superior (IES) cujos pesquisadores mais realizaram publicações sobre gestão de estoques foi a Universidade de São Paulo (USP), com 5 artigos publicados entre 2010 e 2018. As IES Universidade Federal da Paraíba (UFPB), Universidade Federal de Pernambuco (UFPE), Universidade de Santa Catarina (UFSC), Universidade Federal de São Carlos (UFSCAR), Universidade Federal de Uberlândia (UFU), Universidade Metodista de Piracicaba (UNIMEP) e Universidade de Brasília (UNB) realizaram a mesma quantidade de publicações (2) no período analisado.

Esses resultados indicam que a produção sobre o tema se concentra em universidades públicas, que são responsáveis pela maior parte das publicações nacionais. Além disso, a IES Washburm University, localizada no estado de Kansas, EUA, foi a única universidade internacional que realizou trabalhos sobre gestão de estoques como autor ou coautor de artigos publicados no Brasil. Com isso, percebe-se 
uma carência de parcerias internacionais em publicações que abrangem a temática, sendo que essas parcerias poderiam estimular inovações em gestão de estoques.

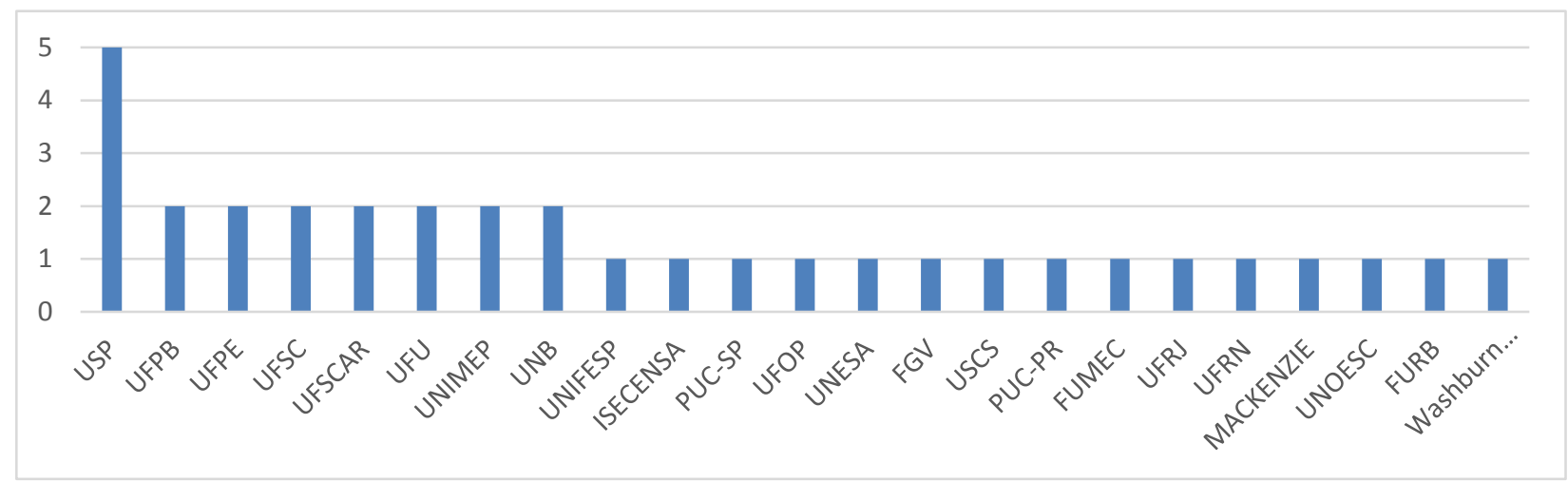

Figura 5. Publicações por Instituição do Ensino Superior (IES)

Fonte: Dados da pesquisa.

Os periódicos que mais publicaram sobre gestão de estoques no período de análise foram a Revista Catarinense da Ciência Contábil e Gestão \& Regionalidade. Ambos as revistas tiveram 2 publicações entre 2010 e 2018 e possuem classificação B2 pela Qualis/Capes na área de Administração Pública e de Empresas, Ciências Contábeis e Turismo. Os demais periódicos estão listados na Figura 6 e cada um realizou 1 publicação no período. Esse resultado confirma a Lei de Bradford no que tange à concentração de publicações de artigos relevantes, sobre determinado tema, em um menor número de periódicos, conforme apontado por Araújo (2006). 
Inovação em Logística e Operações: Um Estudo Biblimétrico sobre Gestão de Estoques no Brasil Arthur Antonio Silva Rosa, Thalita Emanuelle Farias Bastos, Brunno da Silva, Kárem Cristina de Sousa

Ribeiro

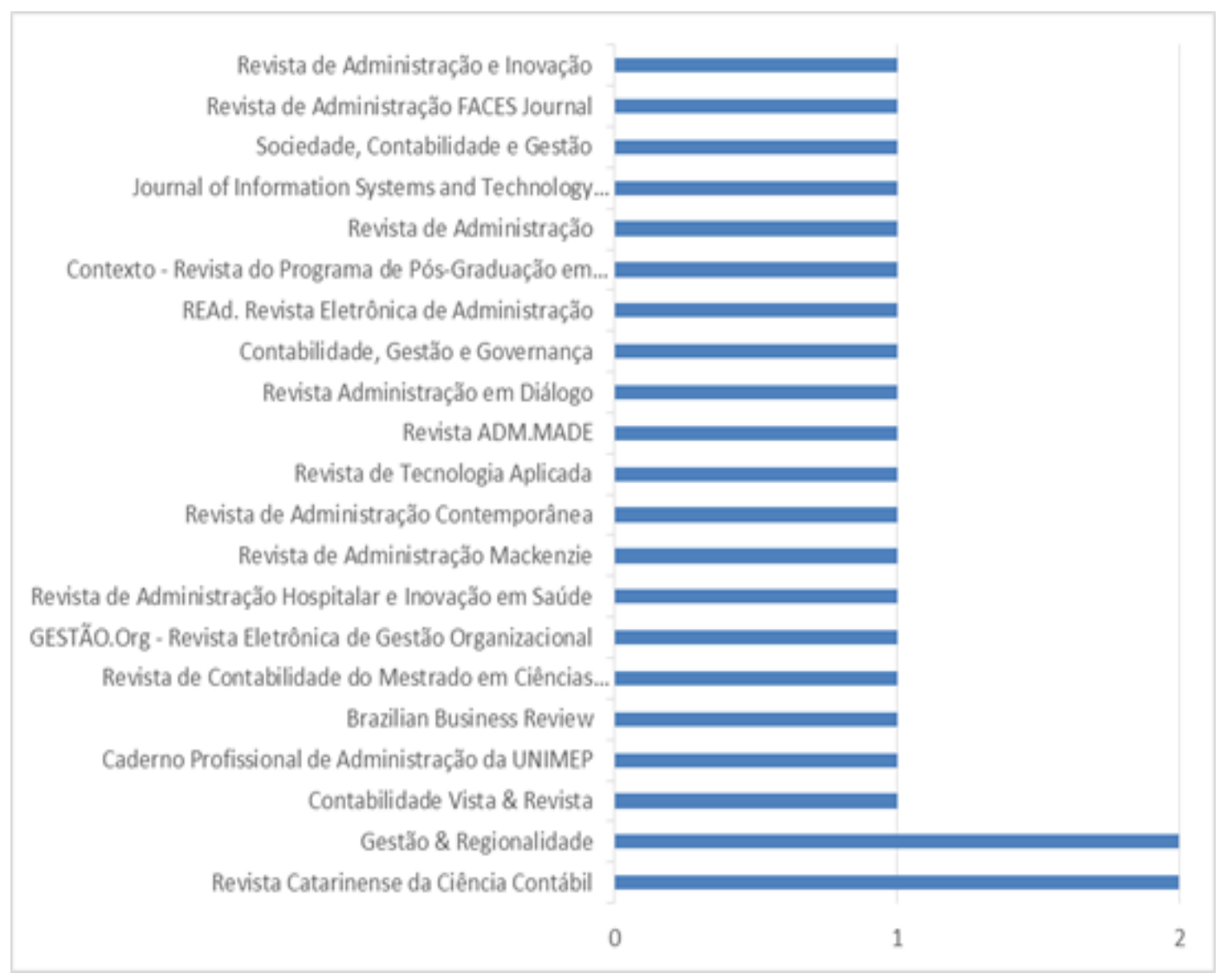

Figura 6. Quantidade de publicações por periódico

Fonte: Dados da pesquisa.

Com base na Figura 7, os métodos de gestão de estoques mais utilizados pelos artigos publicados entre 2010 e 2018 foram o LEC, Just in time e Curva ABC, cada um desses métodos foram utilizados por 3 pesquisas, confirmando a definição dos métodos mais utilizados na gestão de estoque e citado por Scherr (1989), Weston e Brigham (2000), Hansen e Mowen (2001), Assaf Neto (2006), Ballou (2006), Assaf Neto e Silva (2007). 


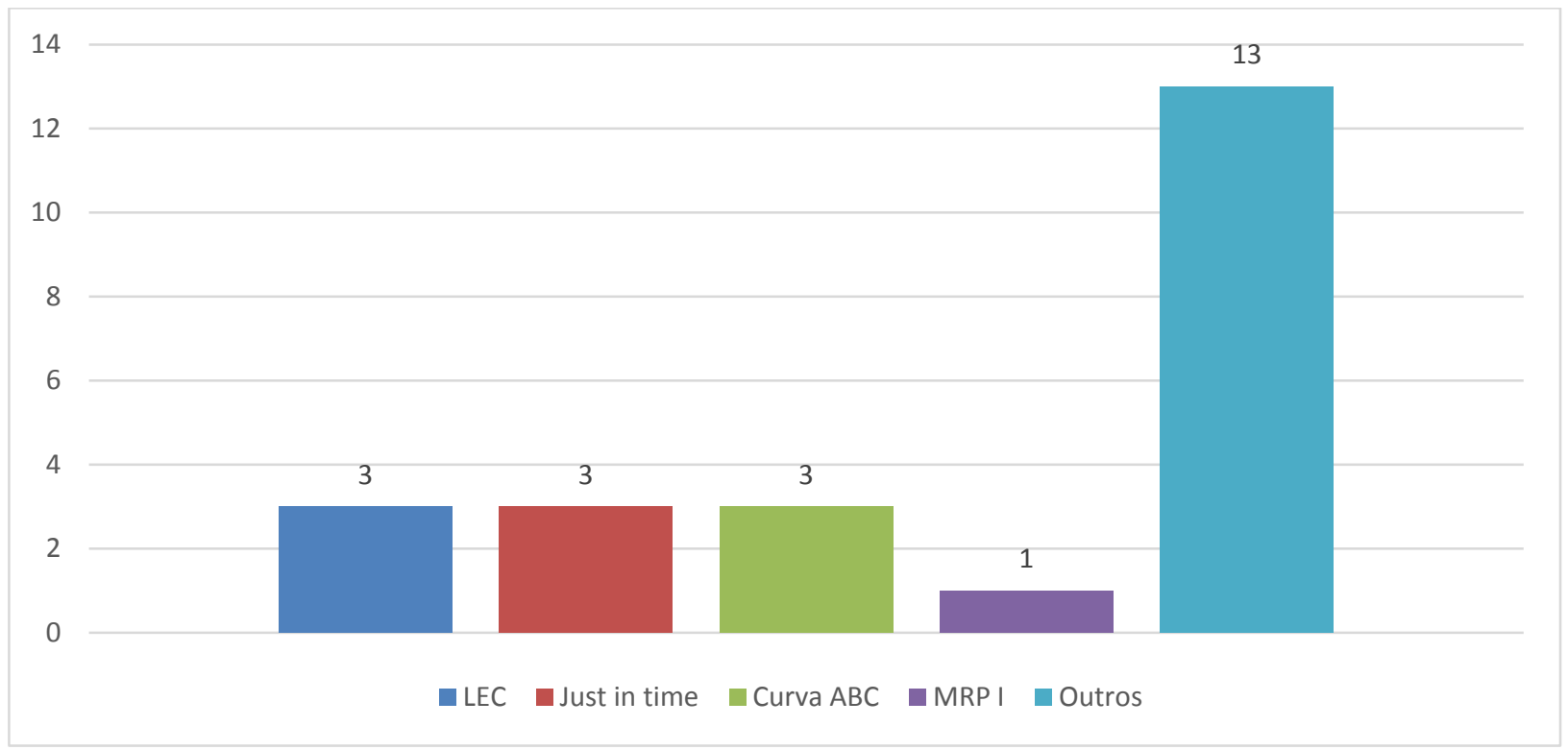

Figura 7. Métodos de gestão de estoques

Fonte: Dados da pesquisa.

Já o método MRP I foi utilizado por apenas 1 artigo e o método MRP II não foi utilizado entre os artigos analisados. Salienta-se que esse último método, especialmente, demanda um investimento significativo em tecnologia visto que é um sistema de informações abrangendo toda a manufatura (Adam \& Cox, 1985). Na categoria "Outros", foram considerados os artigos que não possuem um ou mais métodos específicos em seu estudo no contexto da gestão de estoques.

A Figura 8 apresenta os artigos que foram utilizados no estudo, se os mesmos abordaram inovação na gestão de estoques e a justificativa para tal classificação, respectivamente. A classificação de inovação levou em consideração a utilização de algum método de forma diferente do habitual, seja com uso de alguma ferramenta, cálculo para definição, dentre outros. 


\begin{tabular}{|c|c|c|c|}
\hline Título & Autores/Ano & Inovação & Justificativa \\
\hline $\begin{array}{l}\text { Decisões financeiras de curto prazo das } \\
\text { pequenas e médias empresas industriais: um } \\
\text { estudo exploratório }\end{array}$ & $\begin{array}{l}\text { Márcio André Veras Machado e } \\
\text { Kelly Nayane Brilhante Barreto } \\
\text { (2010) }\end{array}$ & Não & $\begin{array}{l}\text { Descrição das decisões financeiras de curto prazo das pequenas e } \\
\text { médias empresas industriais da cidade de João Pessoa/PB. Em relação } \\
\text { ao estoque, verificou-se que a técnica mais utilizada foi o just in time e } \\
\text { as variáveis mais importantes na avaliação do investimento em estoques } \\
\text { foram a disponibilidade de recursos e os descontos obtidos }\end{array}$ \\
\hline $\begin{array}{l}\text { Políticas e instrumentos gerenciais utilizados na } \\
\text { gestão do capital de giro das pequenas e médias } \\
\text { empresas: um estudo exploratório }\end{array}$ & $\begin{array}{l}\text { Márcio André Veras Machado, } \\
\text { Márcia Reis Machado e Kelly } \\
\text { Nayane Brilhante Barreto }(2010)\end{array}$ & Não & Foco em capital de giro, apenas aborda o controle de estoque \\
\hline $\begin{array}{l}\text { Aplicação do algoritmo genético na gestão de } \\
\text { suprimentos }\end{array}$ & $\begin{array}{l}\text { Roberto Giro Moori, Herbert } \\
\text { Kimura e Oscar Kenjiro Asakura } \\
\text { (2010) }\end{array}$ & Sim & $\begin{array}{l}\text { Aplicação do algoritmo genético como instrumento de tomada decisão } \\
\text { para a gestão de suprimentos }\end{array}$ \\
\hline $\begin{array}{l}\text { O E-SCM e a gestão dos estoques: um estudo de } \\
\text { múltiplos casos em um segmento de cadeia de } \\
\text { lojas de departamento }\end{array}$ & $\begin{array}{l}\text { Alexandre Pereira Salgado } \\
\text { Junior, Juliana Chiaretti Novi, } \\
\text { Antonio Carlos Pacagnella } \\
\text { Junior e Marcio Mattos Borges } \\
\text { de Oliveira (2011) }\end{array}$ & Sim & $\begin{array}{l}\text { Analisou como o e-SCM pode colaborar na manutenção de níveis } \\
\text { adequados de estoques e interrupções nas cadeias de suprimentos. } \\
\text { (O E-SCM é uma nova solução que disponibiliza informações de } \\
\text { consumo em tempo real, sendo mais dinâmica e eficiente que o } \\
\text { tradicional. Dessa forma, melhora ainda mais o nível de serviços } \\
\text { prestados ao consumidor final) }\end{array}$ \\
\hline Gestão estratégica dos estoques & $\begin{array}{l}\text { Irineu Gustavo Nogueira Gianesi } \\
\text { e } \\
\text { Jorge Luiz de Biazzi (2011) }\end{array}$ & Não & $\begin{array}{l}\text { Apresenta a necessidade de as empresas terem uma visão estratégica e } \\
\text { sistêmica na gestão de estoques }\end{array}$ \\
\hline $\begin{array}{l}\text { Planejamento de cenários e amplificação da } \\
\text { variabilidade da demanda }\end{array}$ & $\begin{array}{l}\text { Marina Dais de Faria e José } \\
\text { Luis Felicio dos Santos de } \\
\text { Carvalho (2011) }\end{array}$ & Não & $\begin{array}{l}\text { Utiliza um estudo de caso (ideias de incerteza, transformação, } \\
\text { organizacionação e integração de gestão de estoque) para } \\
\text { aproveitamento em disciplinas de Estratégia, Marketing, Produção e } \\
\text { Ética }\end{array}$ \\
\hline $\begin{array}{l}\text { Proposição de lote econômico como estratégia de } \\
\text { compra de compra para farmácia hospitalar } \\
\text { municipal }\end{array}$ & $\begin{array}{l}\text { Vivian Daronco Cauduro e } \\
\text { Luís Carlos Zucatto (2011) }\end{array}$ & Não & Propõe o LEC para uma farmácia, mas da forma convencional \\
\hline $\begin{array}{l}\text { A gestão da demanda na cadeia de produção da } \\
\text { indústria de laticínios: uma análise dos problemas } \\
\text { e abordagens para melhoria }\end{array}$ & $\begin{array}{l}\text { Daniela de Castro Melo e } \\
\text { Rosane Lúcia Chicarelli } \\
\text { Alcântara (2012) }\end{array}$ & Não & $\begin{array}{l}\text { Identificou os problemas da gestão da demanda na cadeia de } \\
\text { suprimentos do setor de laticínios e propôs abordagens de melhoria } \\
\text { para alcançar a efetividade da gestão da demanda no setor }\end{array}$ \\
\hline $\begin{array}{l}\text { A eficiência da gestão de estoques: estudo sobre } \\
\text { a aplicação do lean manufacturing }\end{array}$ & $\begin{array}{l}\text { Gustavo Benevides, Pedro } \\
\text { Domingos Antoniolli e Ana Rita } \\
\text { Tiradentes Terra Argoud (2013) }\end{array}$ & Sim & $\begin{array}{l}\text { Utilização da tecnologia de rádio frequência (RFID) nos processos } \\
\text { logísticos (oferece informação precisa dos produtos e em tempo real) }\end{array}$ \\
\hline $\begin{array}{l}\text { Práticas logísticas nas pequenas e médias } \\
\text { empresas brasileiras }\end{array}$ & $\begin{array}{l}\text { Juarez Nuno da Silva e Claudio } \\
\text { Pitassi (2013) }\end{array}$ & Não & $\begin{array}{l}\text { Pesquisa de campo, com abordagem qualitativa e com finalidades } \\
\text { descritivas e explicativas para identificar as práticas logísticas presentes } \\
\text { na gestão de seis PMEs brasileiras que contribuem para melhorar o seu } \\
\text { desempenho }\end{array}$ \\
\hline $\begin{array}{l}\text { Gestão de estoque e lean manufacturing: estudo } \\
\text { de caso em uma empresa metalúrgica }\end{array}$ & $\begin{array}{l}\text { Ricardo Aurélio Quinhões Pinto, } \\
\text { Ubiratã Tortato, Claudimar } \\
\text { Pereira da Veiga e Anderson } \\
\text { Catapan (2013) }\end{array}$ & Não & $\begin{array}{l}\text { Análise do impacto da filosofia lean aplicada à gestão de estoque em } \\
\text { uma empresa metalúrgica }\end{array}$ \\
\hline $\begin{array}{l}\text { Contabilidade enxuta (lean accounting) na } \\
\text { indústria automobilística: o caso da fiat }\end{array}$ & $\begin{array}{l}\text { Tiago Nascimento Borges } \\
\text { Slavov, Ana Cristina de Faria, } \\
\text { Luiz Carlos Di Serio e Antonio } \\
\text { Nunes Pereira (2013) }\end{array}$ & Não & $\begin{array}{l}\text { Investiga como os princípios da Contabilidade Enxuta são aplicados e } \\
\text { quais informações são geradas por essa filosofia em montadora da Fiat. } \\
\text { São mensurados os custos, desperdícios e perdas incorridos no } \\
\text { processo produtivo, assim como são geradas informações relativas à } \\
\text { gestão dos estoques, visando dar suporte aos gestores em seu processo } \\
\text { de tomada de decisão }\end{array}$ \\
\hline $\begin{array}{l}\text { Como a Gestão de Práticas de Oferta de Crédito } \\
\text { Impacta a de Estoque }\end{array}$ & $\begin{array}{l}\text { Claudinê Jordão Carvalho } \\
\text { (2015) }\end{array}$ & Não & $\begin{array}{l}\text { Análise do impacto da gestão das práticas de oferta de crédito sobre a } \\
\text { de estoques em uma amostra de } 432 \text { micro e pequenas empresas }\end{array}$ \\
\hline $\begin{array}{l}\text { Planejamento e gestão logística de medicamentos } \\
\text { em uma central de abastecimento farmacêutico } \\
\text { hospitalar }\end{array}$ & $\begin{array}{l}\text { Gustavo Luís Meffe Andreoli e } \\
\text { Cleidson Nogueira Dias (2015) }\end{array}$ & Não & $\begin{array}{l}\text { Estudo de caso para aprimoramento da gestão logística de } \\
\text { medicamentos na Central de Abastecimento Farmacêutico (CAF) de um } \\
\text { Hospital situado em Brasília no Distrito Federal. Como resultado, } \\
\text { elaborou-se um plano de ação com procedimentos habituais de gestão } \\
\text { de estoque }\end{array}$ \\
\hline $\begin{array}{l}\text { Do TOC para manufatura enxuta: um estudo de } \\
\text { caso de mudança de gestão da produção }\end{array}$ & $\begin{array}{l}\text { Ricardo Aurélio Quinhões Pinto, } \\
\text { Alvair Silveira Torres Júnior e } \\
\text { Ubiratã Tortato (2015) }\end{array}$ & Não & $\begin{array}{l}\text { Pesquisa exploratória descritiva sobre uma empresa que trocou a forma } \\
\text { de gestão da produção de Teoria das Restrições (TOC) para manufatura } \\
\text { enxuta }\end{array}$ \\
\hline $\begin{array}{l}\text { Praticando gestão de operações em um } \\
\text { laboratório de gestão }\end{array}$ & $\begin{array}{l}\text { Roberto Portes Ribeiro, Antonio } \\
\text { Carlos Aidar Sauaia, Adriana } \\
\text { Marotti de Mello e Alvair Silveira } \\
\text { Torres Júnior (2015) }\end{array}$ & Não & $\begin{array}{l}\text { Análise descritiva do laboratório de gestão, na qual se adotou o método } \\
\text { de estudo de caso longitudinal, com abordagem quantitativa, na } \\
\text { perspectiva de uma pesquisa bibliométrica }\end{array}$ \\
\hline $\begin{array}{l}\text { Impactos da sazonalidade da produção sobre os } \\
\text { estoques e lucratividade: análise do segmento } \\
\text { industrial brasileiro }\end{array}$ & $\begin{array}{l}\text { José Marcos Carvalho de } \\
\text { Mesquita, Henrique Cordeiro } \\
\text { Martins, Alexandre Teixeira Dias } \\
\text { e Aril Rabelo (2016) }\end{array}$ & Não & $\begin{array}{l}\text { Avaliar os efeitos de variações sazonais de produção sobre estoques e } \\
\text { rentabilidade de empresas industriais }\end{array}$ \\
\hline $\begin{array}{l}\text { Adequação das práticas de gestão de estoques: o } \\
\text { caso de uma microempresa do setor de móveis da } \\
\text { zona norte de SP }\end{array}$ & $\begin{array}{l}\text { Juliano de Campos Melo e } \\
\text { André Taue Saito (2016) }\end{array}$ & Não & $\begin{array}{l}\text { Estudo de caso de uma microempresa, que atua no comércio de móveis } \\
\text { para escritório, para identificação das ferramentas/modelos de gestão de } \\
\text { estoque utilizados. Como resultado, verificou-se a utilização de } \\
\text { ferramentas tradicionais }\end{array}$ \\
\hline $\begin{array}{l}\text { Estrutura da tecnologia de informação para a } \\
\text { gestão de demanda da cadeia de suprimento } \\
\text { farmacêutica: um estudo de caso brasileiro }\end{array}$ & $\begin{array}{l}\text { Pedro Domingos Antoniolli } \\
\text { (2016) }\end{array}$ & Sim & $\begin{array}{l}\text { Aplicação de soluções de Tecnologia da Informação em processos de } \\
\text { gestão de demanda farmacêutica }\end{array}$ \\
\hline $\begin{array}{l}\text { Aplicação do lote econômico de compras (LEC): } \\
\text { estudo de caso de uma lanchonete familiar do } \\
\text { município de campos dos Goytacazes/RJ }\end{array}$ & $\begin{array}{l}\text { Rodolpho Rodighiero Pinto e } \\
\text { Leonardo da Silva Ribeiro } \\
(2016)\end{array}$ & Não & $\begin{array}{l}\text { Aplicação do Lote Econômico de Compras (LEC) com o auxílio da curva } \\
\text { ABC e previsão de demanda móvel para uma lanchonete na cidade de } \\
\text { Campos dos Goytacazes/RJ }\end{array}$ \\
\hline $\begin{array}{l}\text { Utilização de ferramentas gerenciais para o } \\
\text { controle de estoques: um estudo de caso de uma } \\
\text { empresa do setor alimentício }\end{array}$ & $\begin{array}{l}\text { Diego Camilo Ferreira Sousa, } \\
\text { Calline Neves de Queiroz } \\
\text { Claudino, Joás Tomaz de } \\
\text { Aquino e Fagner José Coutinho } \\
\text { de Melo (2017) }\end{array}$ & Não & $\begin{array}{l}\text { Analisar o atual sistema de gerenciamento de estoque de uma empresa } \\
\text { do setor alimentício localizada em Pernambuco e propor melhorias a } \\
\text { esse sistema }\end{array}$ \\
\hline $\begin{array}{l}\text { Análise dos critérios utilizados em relação aos } \\
\text { estoques obsoletos em uma empresa atacadista }\end{array}$ & $\begin{array}{l}\text { Emerson Amaro Lemes e } \\
\text { Emanoel Marcos Lima (2017) }\end{array}$ & Não & $\begin{array}{l}\text { Estudo de caso para verificar os procedimentos utilizados por uma } \\
\text { empresa do ramo atacadista, em relação à provisão de estoques } \\
\text { obsoletos e se as regras aplicadas estão de acordo com a legislação } \\
\text { vigente }\end{array}$ \\
\hline $\begin{array}{l}\text { Adaptações necessárias para implantação do } \\
\text { bloco k do sped fiscal nas organizações }\end{array}$ & $\begin{array}{l}\text { Débora Alline de Mello, David } \\
\text { Rodrigo Petry, Jorge Carlos } \\
\text { Paludo e leda Margarete Oro } \\
\text { (2018) }\end{array}$ & Não & $\begin{array}{l}\text { Identificar quais são as principais adaptações administrativas e } \\
\text { gerenciais necessárias para a mudança no sistema de controle dos } \\
\text { estoques de produtos nas empresas, em relação à implantação do Bloco } \\
\text { K no SPED Fiscal }\end{array}$ \\
\hline
\end{tabular}

Figura 8. Inovação e gestão de estoques

Fonte: Dados da pesquisa. 
A Figura 9 apresenta a relação entre a quantidade de artigos que possuem inovação em relação à gestão de estoques e aqueles que não possuem.

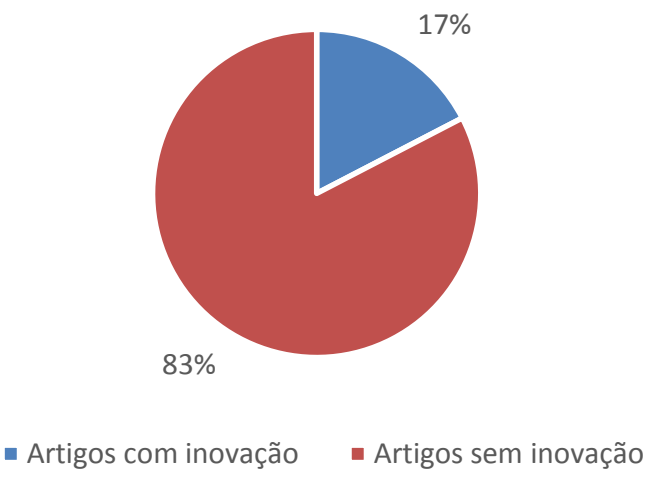

Figura 9. Presença de inovação em gestão de estoques

Fonte: Dados da pesquisa.

Percebe-se que $83 \%$ dos artigos analisados no período não apresentam inovação, indicando uma grande lacuna para futuras pesquisas sobre a temática de gestão de estoques. E observa-se que os principais pontos de inovação estão relacionados à Tecnologia da Informação, visando a otimização de processos quanto à gestão de estoques, como por exemplo, a utilização de algoritmo genético e a maior integração entre os membros da cadeia de suprimentos, assim como a automatização de processos de gestão de estoques por meio de softwares.

Esse resultado está de acordo com os trabalhos de Salgado Junior, Novi, Junior, e de Oliveira (2011) e de Benevides, Antoniolli e Argoud (2013) que analisaram como o uso de uma solução que disponibiliza informações de consumo em tempo real pode colaborar na manutenção de níveis adequados de estoques e interrupções nas cadeias de suprimentos. Além disso, os resultados confirmam o estudo de Ye e Ge (2019) que identificaram uma mudança de foco da gestão de estoques no sentido de aplicar a tecnologia aos seus processos, gerenciamento e otimização. 
Inovação em Logística e Operações: Um Estudo Biblimétrico sobre Gestão de Estoques no Brasil

Arthur Antonio Silva Rosa, Thalita Emanuelle Farias Bastos, Brunno da Silva, Kárem Cristina de Sousa

Ribeiro

\section{CONSIDERAÇÕES FINAIS}

O objetivo deste trabalho foi atingido uma vez que foram definidas as principais características das publicações realizadas sobre a gestão de estoques e inovação no período analisado. Os resultados foram apresentados através de uma análise descritiva, mostrando itens como a evolução da produção científica, pesquisadores e veículos de publicação. O ano que mais apresentou publicações sobre o tema foi 2016, com 5 publicações, seguidos por 2011 e 2015. Aproximadamente a metade da quantidade de publicações são compostas por 2 autores e a IES que mais autores estão afiliados foi a USP.

A Revista Catarinense da Ciência Contábil e a Gestão \& Regionalidade foram os periódicos com o maior número de publicações. Quanto aos métodos mais utilizados de gestão de estoques foram Just in time, LEC e Curva ABC. Cerca de $83 \%$ dos artigos não possuem inovação em relação à gestão de estoques.

A principal limitação do trabalho foi a utilização de apenas uma base de dados que pode ter afetado o tamanho da amostra. Quanto às contribuições, o estudo definiu o perfil de publicações sobre gestão de estoques, auxiliando na definição de temas para futuros trabalhos, principalmente no que diz respeito à inovação na gestão de estoques. Para pesquisas futuras, sugere-se a utilização de outras bases de dados e até mesmo realizar um comparativo das publicações realizadas no Brasil com as de outros países.

\section{REFERÊNCIAS}

Araújo, C. A. (2006). Bibliometria: evolução histórica e questões atuais. Em questão, 12(1), 11-32.

Adams, F. P., \& Cox, J. F. (1985). Manufacturing resource planning: An information systems model. Long Range Planning, 18(2), 86-92.

Arnold, J. R. T. (2001). Administração de Materiais: uma introdução. São Paulo, Atlas.

Assaf Neto A. (2006). Finanças corporativa e valor. São Paulo, Atlas. 
Inovação em Logística e Operações: Um Estudo Biblimétrico sobre Gestão de Estoques no Brasil

Arthur Antonio Silva Rosa, Thalita Emanuelle Farias Bastos, Brunno da Silva, Kárem Cristina de Sousa

Ribeiro

Assaf Neto A. \& Silva, C. A. T. (2007). Administração do Capital de Giro. São Paulo, Atlas.

Ballou, R. H. (2006). Gerenciamento da Cadeia de Suprimentos/logística empresarial; tradução Raul Rubenich.

Baregheh, A., Rowley, J., \& Sambrook, S. (2009). Towards a multidisciplinary definition of innovation. Management decision, 47(8), 1323-1339.

Benevides, G., Antoniolli, P. D., \& Argoud, A. R. T. T. (2015). A eficiência da gestão de estoques: estudo sobre a aplicação do lean manufacturing. Revista de Tecnologia Aplicada, 2(2).

Callefi, J. S. (2019). Custo do Estoque para Atendimento da Demanda a partir da Distribuição Normal. ABCustos, 14(1).

Chen, X., Sim, M., Simchi-Levi, D., \& Sun, P. (2007). Risk aversion in inventory management. Operations Research, 55(5), 828-842.

Chesbrough, H. (2010). Business model innovation: opportunities and barriers. Long range planning, 43(2-3), 354-363.

Cohen, W., \& Levinthal, D. (1990). Absorptive Capacity: A New Perspective on Learning and Innovation. Administrative Science Quarterly, 35(1), 128-152.

Corrêa, H. L., Gianesi, I. G., \& Caon, M. (2001). Planejamento, programação e controle da produção. São Paulo: Atlas, 1.

Costa, J. D. C. (2012). Gestão de estoque de materiais de baixíssimo giro considerando processos críticos para a organização.

Davis, M. M., Chase, R. B., \& Aquilano, N. J. (2001). Fundamentos da administração da produção. Bookman.

Ferreira, N. S. A. (2002). As pesquisas denominadas" estado da arte. Educação \& sociedade, 23, 257.

Garcia, E. S., \& Ferreira Filho, V. J. M. (2009). Cálculo do ponto de pedido baseado em previsões de uma política $<\mathrm{Q}, \quad \mathrm{r}>$ de gestão de estoques. Pesquisa Operacional, 29(3), 605-622.

Garrison, R. H.; Noreen, E. W.; Brewer, P. C. (2007). Contabilidade gerencial. Rio de Janeiro, LCT. 
Inovação em Logística e Operações: Um Estudo Biblimétrico sobre Gestão de Estoques no Brasil

Arthur Antonio Silva Rosa, Thalita Emanuelle Farias Bastos, Brunno da Silva, Kárem Cristina de Sousa

Ribeiro

Gianesi, I. G. N., \& de Biazzi, J. L. (2011). Gestão estratégica dos estoques. Revista de Administração, 46(3), 290-304.

Gil, A. C. (2002). Como elaborar projetos de pesquisa. São Paulo, 5(61), 16-17.

Hansen, D. R., Mowen, M. M., \& Taylor, R. B. (2001). Gestão de custos: contabilidade e controle. São Paulo, Cengage Learning.

Herbon, A., Levner, E., \& Cheng, T. C. E. (2014). Perishable inventory management with dynamic pricing using time-temperature indicators linked to automatic detecting devices. International Journal of Production Economics, 147, 605-613.

Koumanakos, D. P. (2008). The effect of inventory management on firm performance. International journal of productivity and performance management, 57(5), 355-369.

Lee, C. K. M., Lv, Y., Ng, K. K. H., Ho, W., \& Choy, K. L. (2018). Design and application of internet of things based warehouse management system for smart logistics. International Journal of Production Research, 56(8), 2753-2768.

Librantz, A. F. H., de Araújo, S. A., Alves, W. A. L., \& Pileggi, G. C. F. (2010). Algoritmos de busca aplicados na estimação de parâmetros em um modelo probabilístico de gestão de estoque. Exacta, 8(2), 237-248.

Longaray, A. A., Tondolo, V. A. G., Ávila, R. L., Munhoz, P. R., Tondolo, R. R. P., Sarquis, A. B. (2017). Práticas de gestão de estoque no varejo: um panorama da produção científica brasileira. Revista Eletrônica Sistemas \& Gestão, 12(1), 477490.

Maçada, A. C. G., Feldens, L. F., \& Santos, A. M. dos. (2007). Impacto da tecnologia da informação na gestão das cadeias de suprimentos: um estudo de casos múltiplos. Revista Gestão \& Produção, 14(1), 1-12.

Moreira, D. A. (2008). Administração da Produção e Operações. São Paulo, Cengage Learning.

Okubo, Y. (1997). Bibliometric Indicators and Analysis of Research Systems: Methods and Examples. OECD Publishing.

Pinto, R. A. Q., Tortato, U., Da Veiga, C. P., \& Catapan, A. (2013). Gestão de estoque e lean manufacturing: estudo de caso em uma empresa metalúrgica. Revista Administração em Diálogo-RAD, 15(1). 
Rogers, P., Ribeiro, K. C. S., \& Rogers, D. (2004). Avaliando o risco na gestão financeira de estoques. Simpósio de Administração da Produção, Logística e Operações Internacionais, 7.

Rosa, H., Mayerle, S. F., \& Gonçalves, M. B. (2010). Controle de estoque por revisão contínua e revisão periódica: uma análise comparativa utilizando simulação. Production, 20(4), 626-638.

Sacomano, J. B. (1990). Uma análise da estrutura funcional do planejamento e controle da produção e suas técnicas auxiliares (Tese de Doutorado). EESC/USP, São Carlos.

Scheidegger, A. P. G.; Favaretto, F.; \& Lima, R. S. (2013, novembro). Gestão de estoques e políticas de reposição: um estudo bibliométrico da produção científica nas bases de dados Web of Science, Scopus e Scielo. Anais do Simpósio de Engenharia e Produção. Bauru, SP, Brasil, 20.

Junior, A. P. S., Novi, J. C., Junior, A. C. P., \& de Oliveira, M. M. B. (2011). O e-SCM ea gestão dos estoques: um estudo de múltiplos casos em um segmento de cadeia de lojas de departamento. JISTEM-Journal of Information Systems and Technology Management (Online), 8(2), 367-388.

Scherr, F. C. (1989). Modern working capital management: text and cases. Prentice Hall.

Schwartz, J. D., \& Rivera, D. E. (2010). A process control approach to tactical inventory management in production-inventory systems. International Journal of Production Economics, 125(1), 111-124.

Silveira, V. C., Eduardo, A. S., Ribeiro, J. S., Chaebo, G., Nóbrega, D. C. (2018). Estudo da gestão de estoques a partir das publicações científicas nos últimos 10 anos. Revista de Administração e Negócios da Amazônia, 10(1), 21-34.

Slack, N., Chambers, S., \& Johnston, R. (2009). Administração da produção (Vol. 747). São Paulo: Atlas.

Spieth, P., Schneckenberg, D., \& Matzler, K. (2016). Exploring the linkage between business model (\&) innovation and the strategy of the firm. $R \& D$ Management, 46(3), 403-413.

Stevenson, W. J. (2001). Administração das operações de produção. Rio de Janeiro, LTC. 
Inovação em Logística e Operações: Um Estudo Biblimétrico sobre Gestão de Estoques no Brasil Arthur Antonio Silva Rosa, Thalita Emanuelle Farias Bastos, Brunno da Silva, Kárem Cristina de Sousa

Ribeiro

Teece, D. J. (2010). Business models, business strategy and innovation. Long range planning, 43(2-3), 172-194.

Tidd, J., \& Bessant, J. R. (2018). Managing innovation: integrating technological, market and organizational change (6a ed.). Hoboken, USA: John Wiley \& Sons.

Ye, Y., \& Ge, Y. (2019). A bibliometric analysis of inventory management research based on knowledge mapping. The Electronic Library.

Weston, J. F., \& Brigham, E. F. (2000). Fundamentos da administração financeira (10a ed.). São Paulo: MAKRON.

Data de Submissão: 03/11/2019

Data de Aceite: 22/09/2020 